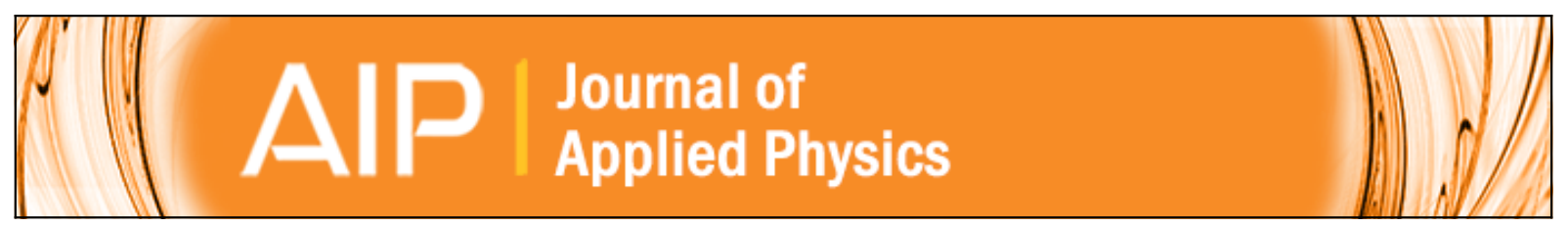

\title{
Bragg band gaps tunability in an homogeneous piezoelectric rod with periodic electrical boundary conditions
}

S. Degraeve, C. Granger, B. Dubus, J. O. Vasseur, M. Pham Thi, and A.-C. Hladky-Hennion

Citation: Journal of Applied Physics 115, 194508 (2014); doi: 10.1063/1.4876757

View online: http://dx.doi.org/10.1063/1.4876757

View Table of Contents: http://scitation.aip.org/content/aip/journal/jap/115/19?ver=pdfcov

Published by the AIP Publishing

\section{Articles you may be interested in}

Symmetry breaking induces band gaps in periodic piezoelectric plates

J. Appl. Phys. 115, 133501 (2014); 10.1063/1.4870137

Piezoelectric and electrostrictive effects in ferroelectret ultrasonic transducers

J. Appl. Phys. 112, 084505 (2012); 10.1063/1.4759052

Lamb wave band gaps in a homogenous plate with periodic tapered surface

J. Appl. Phys. 112, 054503 (2012); 10.1063/1.4749400

Observation of ultrasonic backward beam displacement in transmission through a solid having superimposed periodicity

Appl. Phys. Lett. 97, 041908 (2010); 10.1063/1.3469998

Acoustic band gaps in periodically and quasiperiodically modulated waveguides

J. Appl. Phys. 102, 014902 (2007); 10.1063/1.2749483

\section{AlP Re-register for Table of Content Alerts}




\title{
Bragg band gaps tunability in an homogeneous piezoelectric rod with periodic electrical boundary conditions
}

\author{
S. Degraeve, ${ }^{1}$ C. Granger, ${ }^{1}$ B. Dubus,${ }^{1}$ J. O. Vasseur, ${ }^{2}$ M. Pham Thi, ${ }^{3}$ \\ and A.-C. Hladky-Hennion ${ }^{1}$ \\ ${ }^{1}$ ISEN Department, Institut d'Electronique, de Microélectronique et de Nanotechnologies (UMR CNRS 8520), \\ 41 Boulevard Vauban, 59046 Lille, France \\ ${ }^{2}$ Institut d'Electronique, de Microélectronique et de Nanotechnologies (UMR CNRS 8520), Cité Scientifique, \\ 59652 Villeneuve d'Ascq Cedex, France \\ ${ }^{3}$ THALES Research \& Technology France, Campus Polytechnique, 1 Avenue Augustin Fresnel, \\ 91767 Palaiseau Cedex, France
}

(Received 26 March 2014; accepted 4 May 2014; published online 21 May 2014)

\begin{abstract}
An homogeneous piezoelectric rod is shown to exhibit Bragg band gaps when an electrical boundary condition is applied periodically with the help of metallic electrodes. An analytical model is developed which formulation depends on the applied electric boundary condition and reveals that Bragg band gaps occurring in this very peculiar phononic crystal are related to the electric charge located on the electrodes. Moreover, via an accurate boundary condition (electrodes connected in short circuit, in open circuit, or through an external capacitance), full tunability of the Bragg band gaps can be achieved. Measurements of ultrasonic transmission present an overall excellent agreement with the theoretical results. This phononic crystal can be easily manufactured and presents many potential applications as frequency filters especially for radio frequency telecommunications. (C) 2014 AIP Publishing LLC. [http://dx.doi.org/10.1063/1.4876757]
\end{abstract}

\section{INTRODUCTION}

Phononic crystals (PCs) usually defined as artificial composite materials with a periodic structure have received a great deal of attention during the last two decades. Of particular interest is the existence, due to Bragg scattering, of frequency ranges where the propagation of elastic waves is forbidden. These Bragg band gaps confer to PCs many potential applications as sonic insulators for audible frequencies or efficient filtering devices in various frequency domains, from the $\mathrm{kHz}$ to the $\mathrm{GHz}$, depending upon the order of magnitude of the periodicity. ${ }^{1,2}$

Recent developments on PCs focus on the possibility of enhancing their filtering functionalities by tuning the Bragg stop bands. Main characteristics of these tunable stop band PCs are given in Table I. Tunability can be achieved by modifying the physical properties of the constitutive materials via an external stimulus such as the temperature, ${ }^{3,4}$ an external electric field when one of the components is a electrorheological material, ${ }^{5}$ or an external stress. ${ }^{6}$ With these approaches, limited tunability effect was observed by applying very large amplitude stimuli that can lead to unwanted structural modifications of the PC. Using active materials as PC components constitutes an alternate way for tuning the band gap properties of PCs. It was demonstrated numerically, for example, that the band structure of a PC made of magneto-elastic inclusions embedded in a polymer matrix can be controlled by the application of an external magnetic field. ${ }^{7,8}$ Moreover, several authors have shown that taking into account the piezoelectric effect may modify the dispersion curves of a PC made of one or several piezoelectric materials. ${ }^{9-12} \mathrm{Hsu}^{13}$ reported on band gaps in two-dimensional PC slabs made of ternary lead indium niobate-lead magnesium niobate-lead titanate (PIN-PMN-PT) cylindrical inclusions embedded in epoxy and proved numerically that band gaps tunability can be achieved by changing the electric boundary condition (open-circuit or short circuit) on the top and bottom surfaces of the piezoelectric material. The polymer matrix usually used in such kind of 1-3 composites reduces the effective impedance of the PC but is detrimental for the piezoelectric properties of the overall structure. To increase the control of band gap properties, Chen et al. ${ }^{14}$ have studied elastic wave propagation in beams with periodic array of piezoelectric patches bonded on beam surface and connected to negative capacitance. Very large variations of band gap central frequency and width have been calculated. This result, which requires the stability of the negative capacitance set-up over a wide frequency range, still has to be experimentally confirmed. Some authors ${ }^{15-18}$ have considered electric boundary conditions such as inductance or $R L C$ circuits which introduce additional resonances in the system. Thus, narrow hybridization stop bands appearing in the dispersion curves can be tuned by varying external inductance or capacitance, and narrow pass band can also be inserted inside Bragg band gaps. Finally, some authors have shown the possibility of realizing controllable filtering devices with piezoelectric homogeneous solids by spatially patterning the polarization distribution along one ${ }^{19}$ or two dimensions, ${ }^{20}$ which remains a very challenging topic.

Bragg band gaps are usually produced by wave propagation in periodic structures exhibiting a mechanical impedance mismatch or nonuniform geometry. ${ }^{21}$ They also appear in uniform homogeneous structures submitted to periodical boundary conditions. This problem was extensively studied in the case of elastic waves propagating in beams and plates, ${ }^{2,23}$ showing that change in mechanic boundary conditions could affect strongly Bragg band gaps. Although 
TABLE I. Characteristics of tunable Bragg band gaps in phononic crystals. Initial 1st band gap central frequency is used for normalization.

\begin{tabular}{|c|c|c|c|c|c|c|}
\hline Geometry & $\begin{array}{c}\text { Materials } \\
\text { (matrix/inclusions) }\end{array}$ & $\begin{array}{c}\text { Type } \\
\text { of control }\end{array}$ & $\begin{array}{l}\text { Control range } \\
\text { or type of external } \\
\text { impedance }\end{array}$ & $\begin{array}{c}\text { Variation of 1st } \\
\text { band gap normalized } \\
\text { central frequency }\end{array}$ & $\begin{array}{l}\text { Variation of 1st band } \\
\text { gap normalized } \\
\text { frequency width }(\%)\end{array}$ & References \\
\hline 2D PC & Quartz/void & Temperature & $0-50^{\circ} \mathrm{C}$ & $1-0.998$ & $0.386-0.388$ & 3 \\
\hline 2D PC & Epoxy/electro-rheological material & DC electric field & $0.5-3.5 \mathrm{kV} \mathrm{m}^{-1}$ & $1-0.934$ & $0.638-0.571$ & 5 \\
\hline 2D PC & Elastomer/void & Stress & $92-95 \mathrm{kPa}$ & $1-1.044$ & $0-0.0062$ & 6 \\
\hline 2D PC & Epoxy/Terfenol D & DC magnetic field & $0-20 \mathrm{kOe}$ & $1-1.337$ & $0-0.712$ & 8 \\
\hline 2D PC & PIN-PMN-PT/void & External impedance & $0-+\infty \mathrm{nF}$ & $1-0.988$ & $0.103-0.078$ & 13 \\
\hline $\begin{array}{l}\text { Beam with } \\
\text { piezoelectric patches }\end{array}$ & Epoxy/Lead Zirconate Titanate (PZT) & External impedance & $0-108 \mathrm{nF}$ & $1-1.714$ & $0.286-1.714$ & 14 \\
\hline Uniform rod & PZT & External impedance & $0-+\infty \mathrm{nF}$ & $1-0.833$ & $0-0.333$ & Thiswork \\
\hline
\end{tabular}

many works addressed the tuning of wave propagation with piezoelectric patches modifying boundary conditions (see, for example, Refs. 24 and 25), these works always involved structures with periodical geometry. The use of controllable local boundary conditions to tune Bragg stop bands in uniform homogeneous structures has not been considered yet, to the best of our knowledge.

In this work, a uniform homogeneous piezoelectric rod with periodic electrical boundary conditions is considered. This structure is constituted of identical piezoelectric rods of finite length (assumed larger than their radius) separated periodically by very thin electrodes. As a matter of results, the periodicity of this structure results solely from the electrical boundary condition imposed on the electrodes. An analytical model is developed to calculate dispersion curves for various electrical boundary conditions (open circuit, short circuit, and external capacitance). The variations of Bragg band gap central frequency, Bragg band gap width and effective velocity at low frequency and low wave number by tuning of external capacitance are evaluated. Ultrasonic experiments are also displayed and compared fairly well with theoretical predictions.

\section{MODEL}

The considered piezoelectric structure is depicted in Fig. 1. The identical piezoelectric rods of length $\ell$ are all poled along the $z$ axis in the positive direction and separated with electrodes of negligible thickness. The radius of the rods is assumed to be much smaller than $\ell$ and the structure can be treated as purely one-dimensional, depending on $z$. A simple analytical model is developed, taking into account the electrical boundary condition imposed on the electrodes in order to calculate the dispersion curves of the structure. Three cases are under study in this paper: the electrodes are isolated (open circuit case), connected together (short circuit case) or connected via an external capacitance $C$ (see Fig. 1). The electrodes are assumed thin enough for their mechanical contribution to be neglected. According to Gauss's law, the electrical displacement $D_{3}$ is uniform in each piezoelectric rod. Moreover, the rod radius being small compared to its length, the only significant stress is along the rod length. Therefore, the most convenient choice for independent variables is $D_{3}$ and stress $T_{3}$. The piezoelectric equations are then ${ }^{26}$

$$
\begin{gathered}
S_{3}(z)=s_{33}^{D} T_{3}(z)+g_{33} D_{3}, \\
E_{3}(z)=-g_{33} T_{3}(z)+\beta_{33}^{T} D_{3},
\end{gathered}
$$

where $S_{3}, T_{3}, E_{3}, D_{3}, g_{33}, s_{33}^{D}$, and $\beta_{33}^{T}$ are the mechanical strain, the mechanical stress, the electric field, the electric displacement, the piezoelectric strain constant, the elastic compliance at constant electric displacement, and the dielectric impermeability at constant stress, respectively. The differentiation of Eq. (1a) with respect to $z$ combined with Newton's law leads to the wave equation

$$
\frac{\partial^{2} U(z, t)}{\partial z^{2}}-\rho s_{33}^{D} \frac{\partial^{2} U(z, t)}{\partial t^{2}}=0,
$$

where $\rho$ is the density, $U(z, t)$ is the displacement with $S_{3}(z)=\partial U / \partial z$. Equation (2) shows that wave velocity inside a piezoelectric rod, $V_{L}=1 / \sqrt{\rho s_{33}^{D}}$ is independent of electrical boundary conditions. In the following, harmonic time dependency $\exp (-j \omega t)$, where $\omega$ is the angular frequency, is

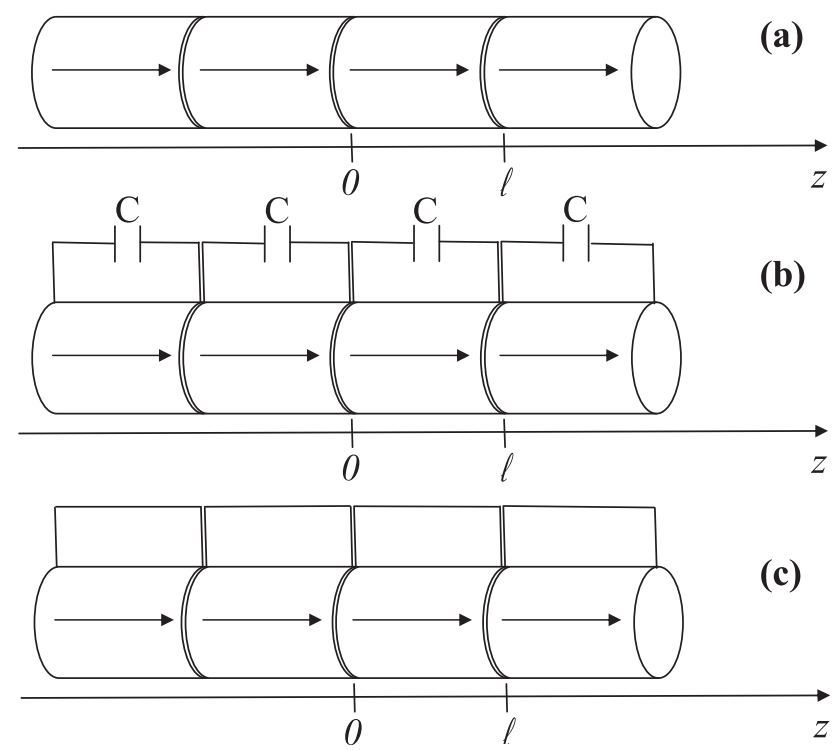

FIG. 1. Schematic description of the PC made of identical piezoelectric rods of length $\ell$, poled along the $z$ axis in the positive direction and separated with electrodes of negligible thickness; (a) all electrodes are in open circuit; (b) electrodes are in short circuit via an external capacitance $C$; and (c) all electrodes are in short circuit. The arrows represent the poling direction. 
assumed for all fields and is omitted in the equations. Inside each layer, the displacement is therefore written as

$$
U(z, t)=A \exp \left(-j k_{1} z\right)+B \exp \left(j k_{1} z\right)
$$

where $k_{1}=\omega / V_{L}$ is the wavenumber associated to the wave propagating inside the rod. Complex coefficients $A$ and $B$ are determined by boundary conditions. As the structure is periodic along $z$ direction due to its periodical electrical boundary condition, the unit cell of length $\ell$ is constituted by one piezoelectric rod. The dispersion relation is obtained by using the Bloch-Floquet relation (BFR) at the interfaces delimiting the unit cell $(z=0$ and $z=\ell$ ). The relation is written for displacement $U$ and stress $T_{3}$ as

$$
\begin{aligned}
U(\ell) & =U(0) \exp (j k \ell), \\
T_{3}(\ell) & =T_{3}(0) \exp (j k \ell),
\end{aligned}
$$

where $k$ is the effective wavenumber associated to the wave propagating in the PC. For the sake of simplicity, we denote $X=\exp (j k \ell)$ in what follows. BFR applied to the displacement combined with Eq. (3) leads to a first relationship between $A$ and $B$

$$
A\left[\exp \left(-j k_{1} \ell\right)-X\right]+B\left[\exp \left(j k_{1} \ell\right)-X\right]=0 .
$$

Similarly, BFR applied to the stress is combined with Eqs. (1) and (3) to give

$$
-A\left[\exp \left(-j k_{1} \ell\right)-X\right]+B\left[\exp \left(j k_{1} \ell\right)-X\right]=\frac{g_{33} D_{3}(1-X)}{j k_{1}} .
$$

It can be noted that, in Eq. (1), at $z=0$ and $z=\ell, T_{3}$ is continuous while $D_{3}$ and $S_{3}$ can be discontinuous if the electric charge on the electrode is not zero. Moreover, $D_{3}$ is uniform in each piezoelectric rod because of Gauss's law, and $D_{3}(z)$ is discontinuous from one rod to the adjacent one. An additional relationship prescribing the electrical boundary conditions on the electrodes is required, together with Eqs. (5) and (6), to get the dispersion relation.

For PC with open circuit electrodes (see Fig. 1(a)), total electrical charge on each electrode is equal to 0. Thus, $D_{3}=0$ and non-trivial solutions of the set of Eqs. (5) and (6) are given by

$$
\left[\exp \left(-j k_{1} \ell\right)-X\right] \cdot\left[\exp \left(j k_{1} \ell\right)-X\right]=0 .
$$

Simple arithmetic leads to solution $k= \pm k_{1}$ showing that wave propagation is dispersionless in that configuration. By inserting these solutions in Eq. (5), it results that $A=0$ or $B=0$. Purely propagative solutions are obtained for $U$, and no reflection takes place at the electrodes.

For PC having all electrodes connected via a capacitance $C$ (see Fig. 1(b)), the line integral of the electric field $E_{3}$ (Eq. (1)) along one rod length is related to the external capacitance $C$, leading to an electric potential $V$ between successive electrodes as

$$
\begin{aligned}
V & =-\int_{0}^{\ell} E_{3} \mathrm{~d} z=\frac{S D_{3}}{C} \\
& =\frac{g_{33}}{s_{33}^{D}}[U(\ell)-U(0)]+\left(\beta_{33}^{T}+\frac{g_{33}^{2}}{s_{33}^{D}}\right) \ell D_{3},
\end{aligned}
$$

where Eq. (1a) was used to express $T_{3}$ as a function of the displacement $U$. In Eq. (8), $S$ stands for the cross section of the rod assumed to be much smaller than its side area. Equation (8) gives a relation between the A and B coefficients and the electrical displacement $D_{3}$, which is written as

$$
\frac{\alpha}{\ell g_{33}}\left[A\left(\exp \left(-j k_{1} \ell\right)-1\right)+B\left(\exp \left(j k_{1} \ell\right)-1\right)\right]=D_{3} .
$$

In Eq. (9), $\alpha$ is defined as

$$
\alpha=\frac{k_{33}^{2}}{1+\frac{C_{0}}{C}},
$$

where

$$
k_{33}^{2}=\frac{g_{33}^{2}}{\left(\beta_{33}^{T} s_{33}^{D}+g_{33}^{2}\right)}
$$

is the electromechanical coupling factor that represents the transduction efficiency of the material, ${ }^{26}$ and

$$
C_{0}=\left(1-k_{33}^{2}\right) \frac{S}{\ell \beta_{33}^{T}}
$$

is the clamped capacitance. When the external electrical capacitance $C$ tends towards zero, the electrical boundary condition corresponds to an open circuit and $\alpha=0$. When the external electrical capacitance $C$ tends to infinity, then the electrical boundary condition corresponds to a short circuit and $\alpha=k_{33}^{2}$.

Equation (9) is inserted into Eq. (6) and leads to

$$
\begin{aligned}
& {\left[\left[\exp \left(-j k_{1} \ell\right)-X\right]+\frac{\alpha}{j k_{1} \ell}(1-X)\left[\exp \left(-j k_{1} \ell\right)-1\right]\right] A} \\
& \quad+\left[\left[-\exp \left(j k_{1} \ell\right)+X\right]+\frac{\alpha}{j k_{1} \ell}(1-X)\left[\exp \left(j k_{1} \ell\right)-1\right]\right] B=0 .
\end{aligned}
$$

Equations (5) and (10) constitute a linear set of equations whose non trivial solutions verify the following quadratic equation:

$$
\begin{aligned}
& {\left[1-\alpha \frac{\sin \left(k_{1} \ell\right)}{k_{1} \ell}\right] X^{2}-2\left[\cos \left(k_{1} \ell\right)-\alpha \frac{\sin \left(k_{1} \ell\right)}{k_{1} \ell}\right] X} \\
& \quad+\left[1-\alpha \frac{\sin \left(k_{1} \ell\right)}{k_{1} \ell}\right]=0 .
\end{aligned}
$$

Solutions of Eq. (11) are either real or complex conjugate. Real solutions correspond to stop band, $k$ being complex in this case, with a real part equal to 0 or $\pi / \ell$. Complex conjugate solutions given by 


$$
X=\frac{\cos \left(k_{1} \ell\right)-\alpha \frac{\sin \left(k_{1} \ell\right)}{k_{1} \ell}}{1-\alpha \frac{\sin \left(k_{1} \ell\right)}{k_{1} \ell}} \pm j \frac{\sin \left(k_{1} \ell\right) \sqrt{1-\alpha \frac{\tan \left(k_{1} \ell / 2\right)}{k_{1} \ell / 2}}}{1-\alpha \frac{\sin \left(k_{1} \ell\right)}{k_{1} \ell}}
$$

correspond to pass band with real $k$. The real part of the complex conjugate solution (Eq. (12)) leads to the dispersion relation

$$
\cos (k \ell)=\frac{\cos \left(k_{1} \ell\right)-\alpha \frac{\sin \left(k_{1} \ell\right)}{k_{1} \ell}}{1-\alpha \frac{\sin \left(k_{1} \ell\right)}{k_{1} \ell}} .
$$

Dispersion relation for open circuit condition (i.e., $C=0$ ) can be recovered by setting $\alpha=0$ in Eq. (13). Dispersion relation for short circuit condition (i.e., $C \rightarrow \infty$ ) is obtained by setting $\alpha=k_{33}^{2}$ in Eq. (13). Any positive capacitance $(0<C<+\infty)$ corresponds to an intermediate case such as $0<\alpha<k_{33}^{2}$.

\section{ANALYTICAL DISPERSION CURVES}

Figure 2 displays calculated band structures in the case of cylindrical rods made of a specific type of PZT, namely PZ29 by Ferroperm, ${ }^{27}\left(\ell=10 \mathrm{~mm}, k_{33}=0.77\right)$. Three cases are presented: the electrodes are isolated (open circuit case, Fig. 2(a)), connected via a capacitance which value is $C$ (Fig. 2(b)), and connected together (short circuit case, Fig. 2(c)). In Fig. 2(b), the value of the capacitance $C$ has been chosen, for the sake of simplicity, to be equal to the clamped capacitance $C_{0}$. The open-circuit and short circuit analytical results are compared with finite element (FE) calculations assuming a rod length much larger than its radius and using the ATILA code. ${ }^{28}$ The agreement is perfect between analytical and numerical results, validating the model. When all the electrodes are in open circuit (Fig. 2(a)), the dispersion curve presents, as mentioned previously, one branch with a slope equal to $V_{L}$. This branch is folded artificially due to the fictitious periodicity. When all the electrodes are in short circuit (Fig. 2(c)), the band structure exhibits a band gap in the frequency range $[128,192] \mathrm{kHz}$ at the border of the Brillouin zone $(k \ell=\pi)$. When the electrodes are connected via an external capacitance $C=C_{0}$, the dispersion curves present an intermediate case between the short circuit and the open circuit cases (Fig. 2(b)), with a band gap in the frequency range $[169,192] \mathrm{kHz}$ at the border of Brillouin zone. One notes that by only changing the electrical condition from the open circuit case to the short circuit case, the structure behaves as a switching device.

The frequency limits of the band gap are determined by setting $\cos (k \ell)=-1$ in Eq. (13). A first solution corresponds to $\sin \left(k_{1} \ell\right)=0$, i.e., $k_{1} \ell=(2 m+1) \pi$ (with $\mathrm{m}$ integer). The corresponding frequencies $(192 \mathrm{kHz}$ when $m=0 ; 576 \mathrm{kHz}$ when $m=1$ ) coincide with the first electrical antiresonance frequency $f_{a}$ of the piezoelectric rod, ${ }^{26}$ associated, respectively, to a half wavelength motion and a three half wavelength motion of the rod. This frequency limit is the same whatever the electrical connection is $(0<C<+\infty)$ and corresponds to the upper limit of the band gap. The other frequency limit of the band gap at the border of the Brillouin zone is obtained by solving $\tan \left(k_{1} \ell / 2\right)=k_{1} \ell /(2 \alpha)$. When $\alpha=k_{33}^{2}$ (short circuit case), the equation gives the electrical resonance frequencies of the $\operatorname{rod} f_{r} .{ }^{26}$ When $0<\alpha<k_{33}^{2}$ (connection via a capacitance), the equation gives an
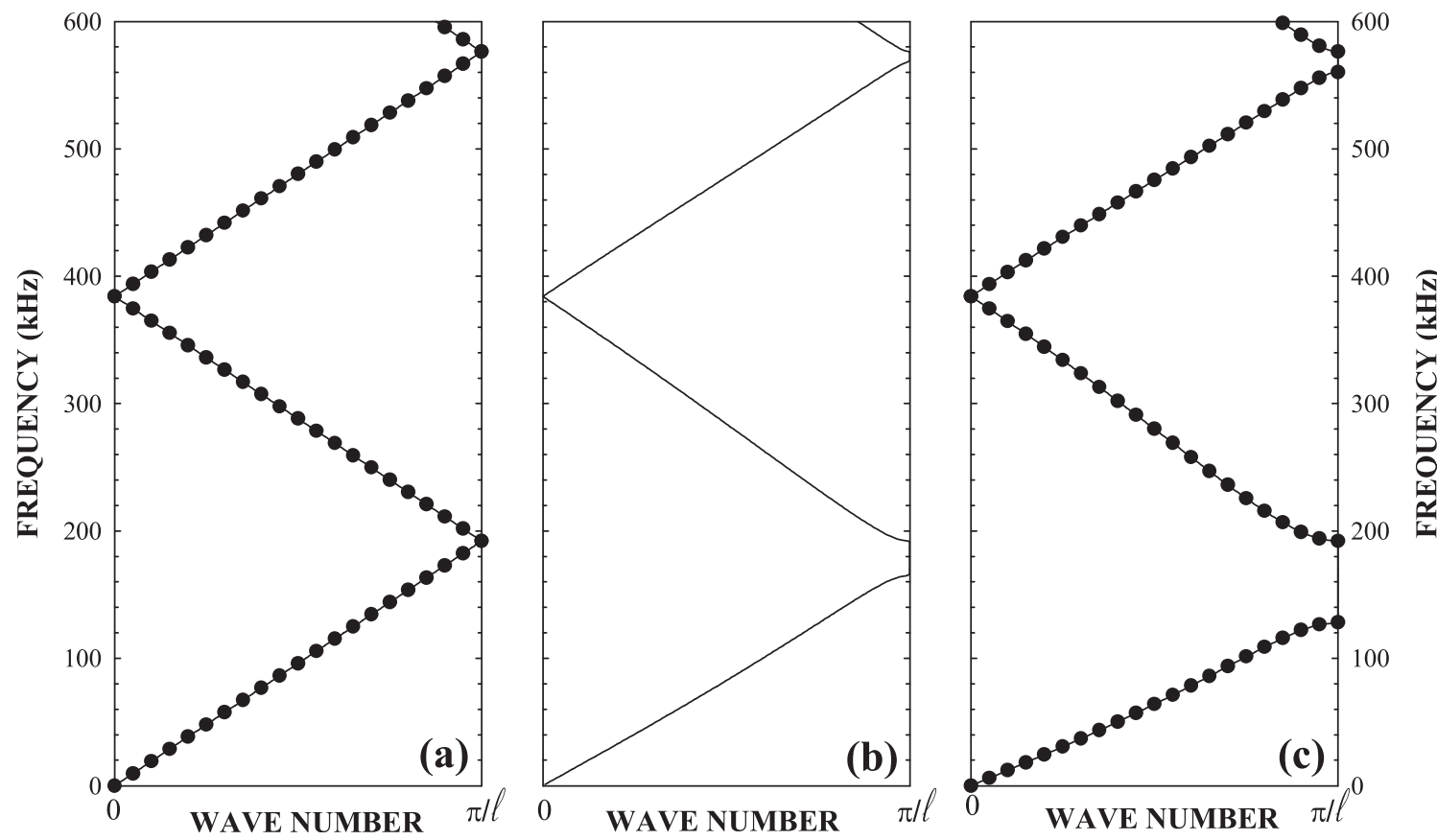

FIG. 2. Analytical dispersion curves of the PC, made of PZ29 rods, $10 \mathrm{~mm}$ long (using Eq. (13)). (a) All electrodes are in open circuit; (b) electrodes are in short circuit via an external capacitance $C$ chosen equals to the clamped capacitance $C_{0}$; and (c) all electrodes are connected together. In (a) and (c), the black dots correspond to the FE results obtained by considering $\ell$ much more larger than the radius of the rods. 
intermediate frequency between the resonance and the antiresonance frequencies $f_{r}$ and $f_{a}$. Thus, by an appropriate choice of the external capacitance $C$, the width of the gap can be tuned: the upper frequency limit is always $f_{a}(192 \mathrm{kHz})$, whereas the lower frequency limit falls in the $\left[f_{r}, f_{a}\right]$ frequency range. The maximal/minimal width of the gap is directly related to the rod electromechanical coupling factor and to the external capacitance $C$. One can notice that the relative width of the first band gap $([128,192] \mathrm{kHz}, 40 \%)$, is greater than the relative width of the second band gap $([560,576] \mathrm{kHz}, 3 \%)$, as the coupling factor $k_{\text {eff }}$ (defined as $\sqrt{1-\left(\frac{f_{r}}{f_{a}}\right)^{2}}$ ) of the three half wavelength mode is smaller than the coupling factor of the half wavelength mode. ${ }^{26}$

In the same way, one can determine the frequency limits of the band gap at the center of the Brillouin zone $(k \ell=0)$ by setting $\cos (k \ell)=1$ in Eq. (13). In that case, only one solution exists, corresponding to $\cos \left(k_{1} \ell\right)=1$. Therefore, no band gap appears at the center of the Brillouin, as observed in Fig. 2.

In the open circuit case, purely propagative solutions (Eq. (7)) are obtained for the displacement, as no reflection takes place $(A=0$ or $B=0)$ in the structure. In the other case (non zero capacitance), by combining Eqs. (5) and (12), a complex relationship between $A$ and $B$ is obtained: the solution consists in a combination of two waves (i.e., one propagating along $z$ positive and one along $z$ negative), showing that the electrical connection induces reflections at the boundaries of the unit cell, i.e., at the electrodes. In addition, as BFR is verified by displacement (Eq. (4)) and because the electric displacement is expressed as a function of the elastic displacement (Eq. (8)), then electric displacement also verifies BFR. Therefore, electric displacement in successive rods $n$ and $n+1$ are related by a factor $\exp (j k \ell)$. The electric displacement is constant in each rod but is discontinuous through the electrode at $z=n \ell$, which carries a charge density $\sigma_{n}$ defined as

$$
\sigma_{n}=D_{3, n+1}-D_{3, n}=[\exp (j k \ell)-1] D_{3, n},
$$

which also verifies the BFR. It shows that, when electrodes are in short circuit together or connected via a capacitance, the reflected field associated to the band gap generation is induced by the electrical charges appearing on the electrodes.

In the long wavelength limit, a second order Taylor expansion of Eq. (13) gives an effective velocity equal to $V_{e f f}(C)=V_{L} \sqrt{1-\alpha}$. As previously shown in Fig. 2, the slope of the dispersion curve in the low frequency range can be continuously tuned as the capacitance is varying. The effective velocity limits are determined by the electromechanical coupling factor and by the external capacitance. In the example presented in this section, the range of variations of the effective velocity is $\left[0.63 V_{L}, V_{L}\right]$. Thus, the performances in terms of tunability of the effective velocity are much better than those found in the literature (see Table I). One can notice that the previous example has been presented for $10 \mathrm{~mm}$ long rods. If one over two electrodes are connected together instead of all the electrodes, then the dispersion curve is obtained by setting $\ell=20 \mathrm{~mm}$ in Eq. (13) instead of $\ell=10 \mathrm{~mm}$. In that case, the band gap appears in the $[64,86] \mathrm{kHz}$ frequency range. In the same way, if one over three electrodes are connected together, then the dispersion curve is obtained by setting $\ell=30 \mathrm{~mm}$ in Eq. (13) and the band gap appears in the [43, 64] kHz. It means that, with the same device, band gap may appear in different frequency ranges, depending on the periodicity of the electrodes used for the electrical connection. Larger is this periodicity, lower is the Bragg band gap frequency domain.

\section{FINITE SAMPLE: EXPERIMENTAL AND NUMERICAL RESULTS}

In order to check the validity of the theoretical model, experiments have been performed with 14 PZ29 rods $(10 \mathrm{~mm}$ long and $5 \mathrm{~mm}$ in radius). Rods are glued with a small amount of epoxy and separated by $25 \mu \mathrm{m}$ thin brass electrodes. The transmission of longitudinal waves through this finite PC is measured using a pair of transducers (Panametrics V101, 0.5 MHz, 1" diameter) and is presented in Fig. 3. The emitter transducer is

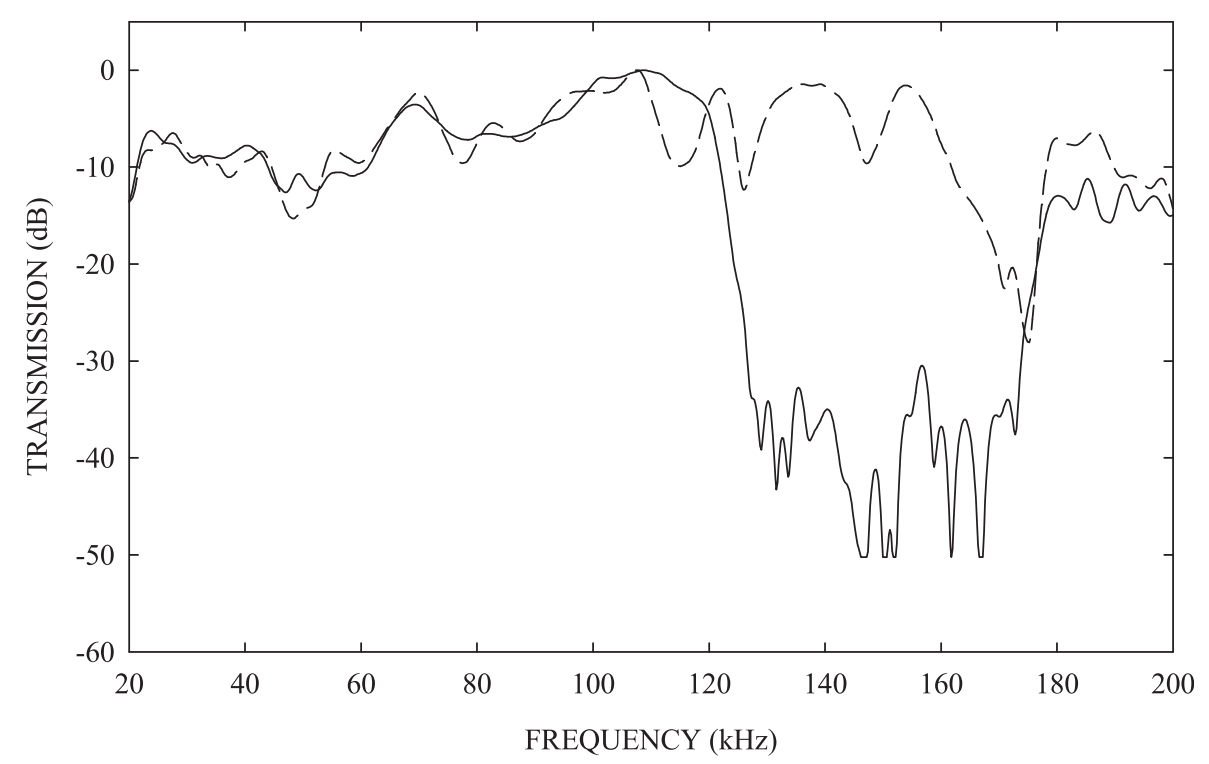

FIG. 3. Experimental transmission (in arbitrary $\mathrm{dB}$ units) through a structure made of 14 PZ29 rods of length $10 \mathrm{~mm}$, and of radius equals to $5 \mathrm{~mm}$. Dashed line: all electrodes are in open circuit, solid line: all electrodes are in short circuit (see Fig. 1). 
excited with a short electric pulse of duration $2 \mu \mathrm{s}$. The received electric signals are averaged over 128 data acquisitions. The transmission spectra are estimated in the spectral domain with the help of a fast Fourier transform of the temporal signals, in the cases of electrodes in open circuit and in short circuit. When the electrodes are unconnected, the transmission exhibits several peaks, related to an integer number of half-wavelength modes, due to the finite size of the PC. To increase the signal to noise ratio in the short circuit case, all electrodes are grounded. This change does not imply any modification in the previous analytical model. In that case, a band gap clearly occurs in the expected frequency range, corresponding to a transmission $30 \mathrm{~dB}$ lower with respect to the unconnected case. Experimental frequency range of the stop band agrees well with the predicted one, although experimentally, the radius is not much smaller than the length. Moreover, glue and electrodes layers are not taken into account in the analytical model. One can notice that in the unconnected case, a parasitic dip appears around $180 \mathrm{kHz}$ : it is due to the radial mode of the rod that appears near the antiresonance frequency of the rod and that has not been taken into account in the analytical model. In order to check this assertion, Fig. 4 presents the analytical dispersion curves as previously, compared to the dispersion curves calculated with the FE method when the exact geometry is taken into account (short circuit and open circuit). It shows the limit of the approximation of the $1 \mathrm{D}$ model as branches related to the radius mode appear around $200 \mathrm{kHz}$. Therefore, the parasitic dip around $180 \mathrm{kHz}$ is well related to the radial mode. Nevertheless, the first band gap is approximately well described by the analytical model. Figure 3 confirms experimentally the existence of the band gap related to the electric displacement discontinuity in the PC and shows unambiguously that this simple structure made of a single piezoelectric material presents a very easily controllable switching functionality in some specific frequency range that can be tuned by changing the length of the connected rods.

Finally, numerical simulations have been performed in order to calculate the transmission, when a longitudinal force is applied at one end of the sample. The FE method is used and the harmonic analysis gives the displacement at each node of the finite element mesh for each frequency given in the calculation. Thanks to the axisymmetrical axis of the sample, the mesh is limited to the half section. In the measurements, the experimental power spectrum is defined as the square of the Fourier transform of the signal measured by the receiver. For a linear approximation, the pressure is proportional to the frequency multiplied by displacement along the sample in the case of harmonic displacement. Therefore, the numerical variations of frequency times displacement are plotted in Fig. 5 for a better comparison with experimental results that measure the acoustic pressure. Numerical results are also normalized and clearly show several peaks at nearly the same position in frequency than the experimental spectrum. One can notice that the numerical peaks are sharper than the experimental ones because in the numerical calculation, the losses are not taken into account. Small discrepancies in the location of the peaks are probably due to the mechanical coupling between the piezoelectric rods that is assumed to be identical in the numerical model, and due to the electrodes that are not taken into account in the modelling. On the transmission curve, the first peak corresponds to a half
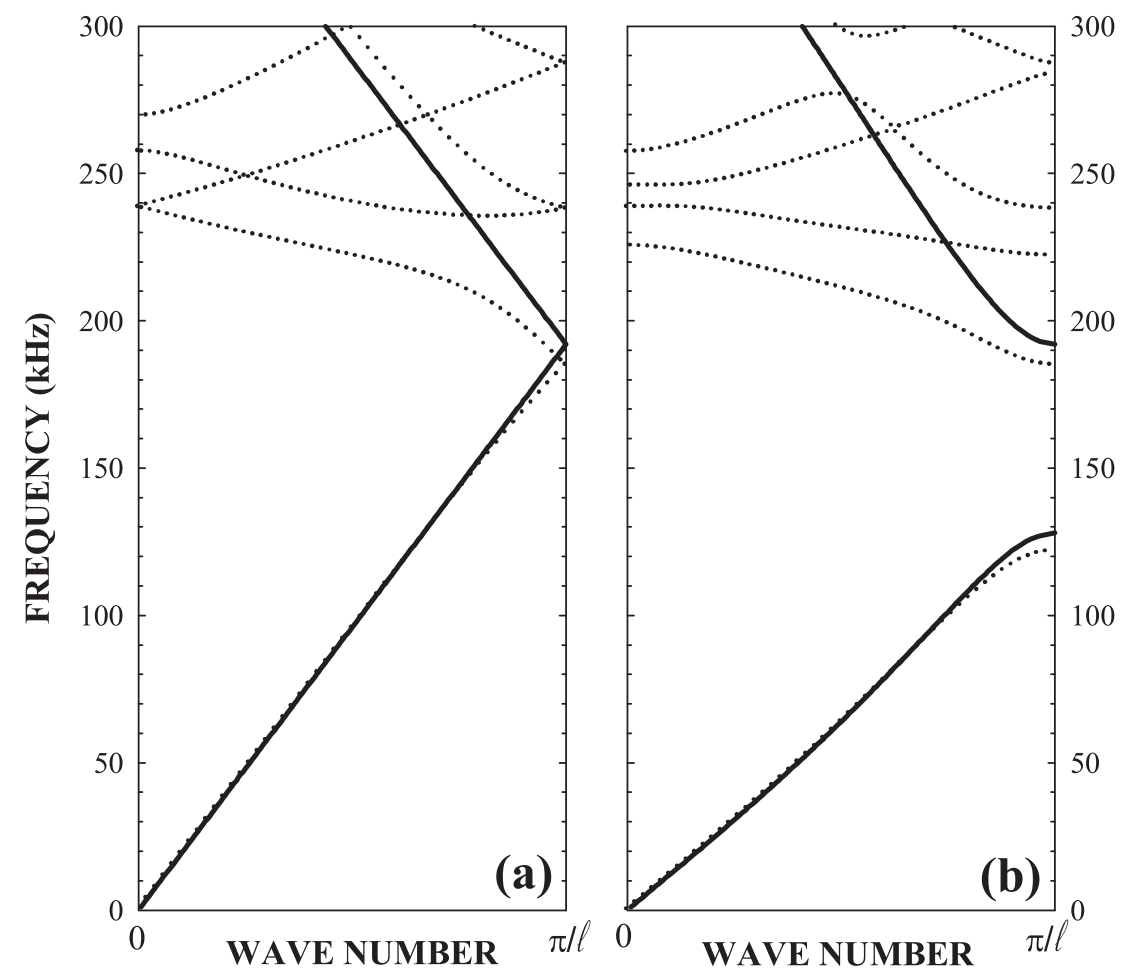

FIG. 4. Dispersion curves of the PC, made of PZ29 rods; (a) all electrodes are in open circuit; (b) all electrodes are in short circuit. In (a) and (b), the solid lines represent the dispersion curves derived from the analytical model with a rod length equals to $10 \mathrm{~mm}$ and neglecting its radius (using Eq. (13)) whereas the dots stand for the FE results considering the actual geometry of the rod (length $=10 \mathrm{~mm}$, radius $=5 \mathrm{~mm}$ ). 


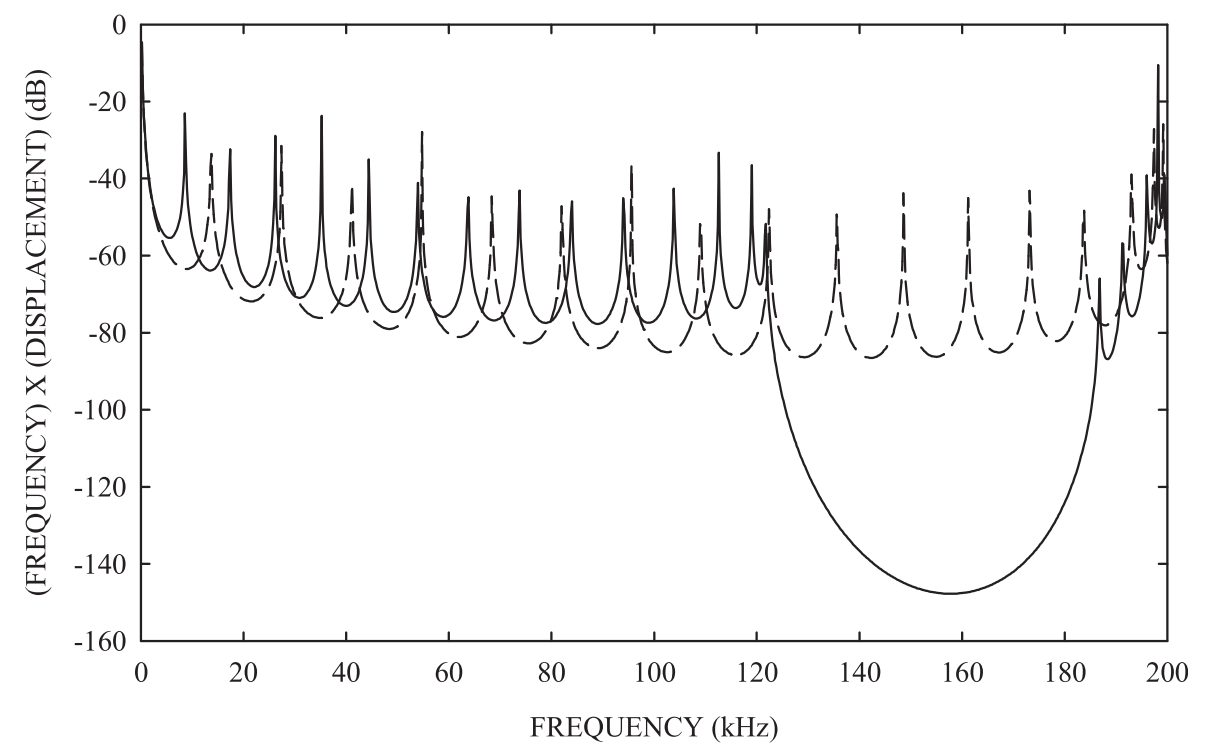

FIG. 5. FE numerical transmission (in arbitrary $d B$ units) through a structure made of 14 PZ29 rods of length and radius equal to $10 \mathrm{~mm}$ and $5 \mathrm{~mm}$, respectively. Dashed line: all electrodes are in open circuit, solid line: all electrodes are in short circuit.

wavelength mode of the whole sample. Their positions are, respectively, $f_{1}(O C)=13.8 \mathrm{kHz} \quad$ and $f_{1}(S C)=8.6 \mathrm{kHz}$ for the open circuit and short circuit cases, respectively. The position of this peak is mainly affected by the effective velocity in the large wavelength limit. As expected, the relative frequency difference between these peaks defined as $\left(f_{1}(O C)-f_{1}(S C)\right) / f_{1}(O C)=37 \%$ is very close to the relative effective velocity difference defined as $\left(V_{\text {eff }}(O C)-V_{\text {eff }}(S C)\right) /$ $V_{\text {eff }}(O C)=36 \%$.

\section{CONCLUSION}

In this paper, an analytical model has been developed for calculating dispersion curves of one dimensional phononic crystals containing rods made of a single piezoelectric material and separated with very thin electrodes. It has been shown that a band gap may appear, depending on the electric boundary condition. When all the piezoelectric rods are connected together or connected via an external capacitance, the band gap is induced by the electric charges appearing on the electrodes. Experiments confirm unambiguously the existence of this band gap. The considered geometry provides numerous possibilities for controlling wave propagation in piezoelectric rods with external variable capacitances:

- Broad tuning of Bragg stop band: relative variation of 1st band gap central frequency $(17 \%)$ and width (from $0 \%$ to $33 \%$ ) with simple variable capacitances;

- Electrical switching between nondispersive and dispersive wave propagation with Bragg band gaps;

- $37 \%$ tuning of effective wave velocity at low frequency and low wave number.

The 1D analytical model presented above can be easily generalised to PCs which unit cell contains one or several piezoelectric materials, with the same poling direction or alternately reverse poling directions. In that case, the dispersion curves may exhibit mixed band gaps, some of them associated with the electrical boundary conditions, others due to classical impedance mismatch. These configurations broaden the designer's possibilities, in order to get a band gap in a given frequency range.

\section{ACKNOWLEDGMENTS}

This work is subject to a THALES/CNRS patent (French Patent Brevet No. 1202561, PCT/EP2013/0070052) and was partially supported by the Agence Nationale de la Recherche with the MIRAGES Project ANR-12-BS09-0015 and the région Nord Pas-de-Calais, France.

${ }^{1}$ Y. Pennec, J. O. Vasseur, B. Djafari-Rouhani, L. Dobrzyński, and P. A. Deymier, Surf. Sci. Rep. 65, 229 (2010).

${ }^{2}$ Acoustic Metamaterials and Phononic Crystals, Springer Series in SolidState Sciences Vol. 173, edited by P. A. Deymier (Springer, Berlin, 2013).

${ }^{3}$ Z.-G. Huang and T.-T. Wu, IEEE Trans. Ultrason. Ferroelectr. Freq. Control 52, 365 (2005).

${ }^{4}$ K. L. Jim, C. W. Leung, S. T. Lau, S. H. Choy, and H. L. W. Chan, Appl. Phys. Lett. 94, 193501 (2009).

${ }^{5}$ J.-Y. Yeh, Physica B 400, 137 (2007).

${ }^{6}$ K. Bertoldi and M. C. Boyce, Phys. Rev. B 77, 052105 (2008).

${ }^{7}$ J.-F. Robillard, O. Bou Matar, J. O. Vasseur, P. A. Deymier, M. Stippinger, A.-C. Hladky-Hennion, Y. Pennec, and B. Djafari-Rouhani, Appl. Phys. Lett. 95, 124104 (2009).

${ }^{8}$ O. Bou Matar, J. F. Robillard, J. O. Vasseur, A.-C. Hladky-Hennion, P. A. Deymier, P. Pernod, and V. Preobrazhensky, J. Appl. Phys. 111, 054901 (2012).

${ }^{9}$ Z. Hou, F. Wu, and Y. Liu, Solid State Commun. 130, 745 (2004).

${ }^{10}$ Y. Wang, F. Li, Y. Wang, K. Kishimoto, and W. Huang, Acta Mech. Sin. 25, 65 (2009).

${ }^{11}$ K. B. Ghazarian and D. G. Piliposyan, J. Sound Vib. 330, 6456 (2011).

${ }^{12}$ D. G. Piliposyan, K. B. Ghazarian, G. T. Piliposian, and A. S. Avertisyan, in Proceedings of ASME 2012 Conference on Smart Materials, Adaptive Structures and Intelligent Systems, SMASIS, Stone Mountain, USA (2012), Vol. 7911, p. 1.

${ }^{13}$ J.-C. Hsu, Jpn. J. Appl. Phys., Part 1 51, 07 GA04 (2012).

${ }^{14}$ S.-B. Chen, J.-H. Wen, D. L. Yu, G. Wand, and X.-S. Wen, Chin. Phys. B 20, 014301 (2011).

${ }^{15}$ G. Wang, S.-B. Chen, and J.-H. Wen, Smart Mater. Struct. 20, 015026 (2011).

${ }^{16}$ S.-B. Chen, J.-H. Wen, G. Wang, D. L. Yu, and X.-S. Wen, J. Intell. Mater. Syst. Struct. 23, 1613 (2012).

${ }^{17}$ F. Casadei, T. Delpero, A. Bergamini, P. Ermanni, and M. Ruzzene, J. Appl. Phys. 112, 064902 (2012).

${ }^{18}$ A. Bergamini, T. Delpero, L. De Simoni, L. Di Lillo, M. Ruzzene, and P. Ermanni, Adv. Mater. 26, 1343 (2013). 
${ }^{19}$ I. V. Ostrovskii, V. A. Klymko, and A. B. Nadtochiy, J. Acoust. Soc. Am. 125, EL129 (2009).

${ }^{20}$ C. J. Rupp, M. L. Dunn, and K. Maute, Appl. Phys. Lett. 96, 111902 (2010).

${ }^{21}$ L. Liu and M. I. Hussein, J. Appl. Mech. 79, 011003 (2012).

${ }^{22}$ D. J. Mead, J. Sound Vib. 11, 181 (1970).

${ }^{23}$ M. L. Rummerman, J. Acoust. Soc. Am. 57, 370 (1975).

${ }^{24}$ O. Thorp, M. Ruzzene, and A. Baz, Smart Mater. Struct. 10, 979 (2001).
${ }^{25}$ A. Baz, J. Vib. Acoust. 123, 472 (2001).

${ }^{26} \mathrm{O}$. B. Wilson, Introduction to Theory and Design of Sonar Transducers (Peninsula Publishing, Los Altos, 1988).

${ }^{27}$ The properties of $P Z 29$ are $\rho=7450 \mathrm{~kg} \mathrm{~m}^{-3}, s_{33}^{D}=9.095 \times 10^{-12} \mathrm{~m}^{2} \mathrm{~N}^{-1}$, $g_{33}=2.418 \times 10^{-2} \mathrm{~V} \mathrm{~m} \mathrm{~N}^{-1}, \beta_{33}^{T}=4.206 \times 10^{7} \mathrm{~m} \mathrm{~F}^{-1}$.

${ }^{28}$ ATILA, Finite-Element Software Package for the analysis of 2D and 3D structures based on smart materials. Version 6.0.2 User's Manual; November 2010. 